\title{
Chain Flexibility in Self-Assembled
}

\section{Monolayers Affects Protein Adsorption and}

\section{Surface Hydration, a Molecular Dynamics}

\section{Study, Supporting Information}

\author{
Wesley Beckner, ${ }^{\dagger}$ Yi He, ${ }^{\ddagger}$ and Jim Pfaendtner, ${ }^{*}$ \\ Department of Chemical Engineering, Seattle, Washington 98105, United States, and \\ College of Chemical and Biological Engineering, Zhejiang University, Hangzhou, Zhejiang, \\ 310027, P. R. China \\ E-mail: jpfaendt@uw.edu
}

${ }^{*}$ To whom correspondence should be addressed

${ }^{\dagger}$ University of Washington

†Zhejiang University 
Supporting information contains a diagram of the design flow for the protein + surface simulations, a plot of the RMSDs of the $\mathrm{C} \alpha$ atoms of lysozyme for each simulation, and the square of the displacement of adsorbed protein for each simulation.

\section{Three Simulations (5 ns):}

frozen surfaces were equilibrated in an NVT water box for $5 \mathrm{~ns}$

to allow waters to diffuse between chains

\section{Triplicate Simulations (100 ns) of Six Chain Immobilization Schemes:}

The surface + water + protein systems were used to spawn triplicates of six distinct chain immobilization schemes for a total of 108 NVT simulations
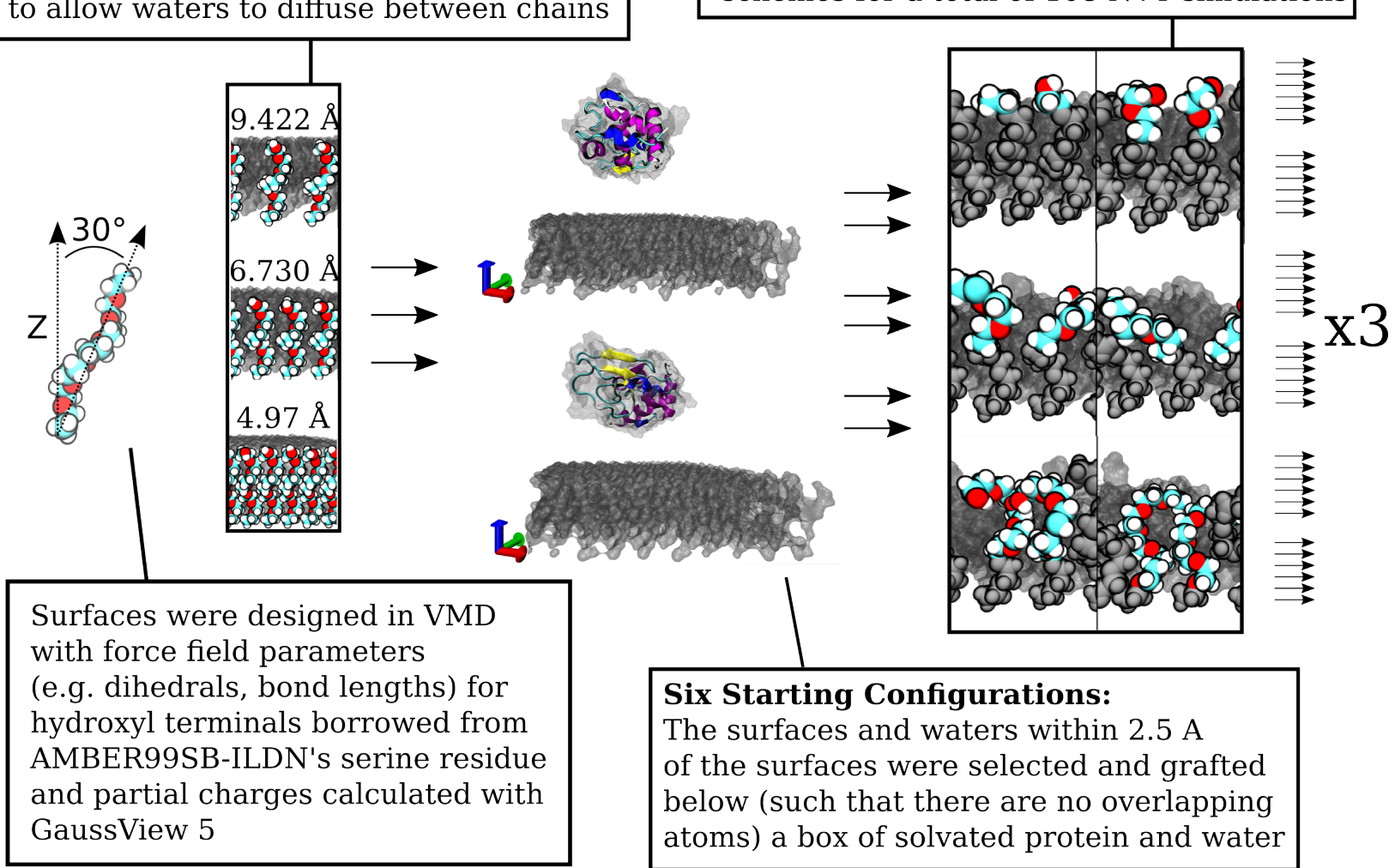

Six Starting Configurations:

The surfaces and waters within $2.5 \mathrm{~A}$ of the surfaces were selected and grafted below (such that there are no overlapping atoms) a box of solvated protein and water

Figure S1: From left to right, design flow for the protein + surface simulations. 

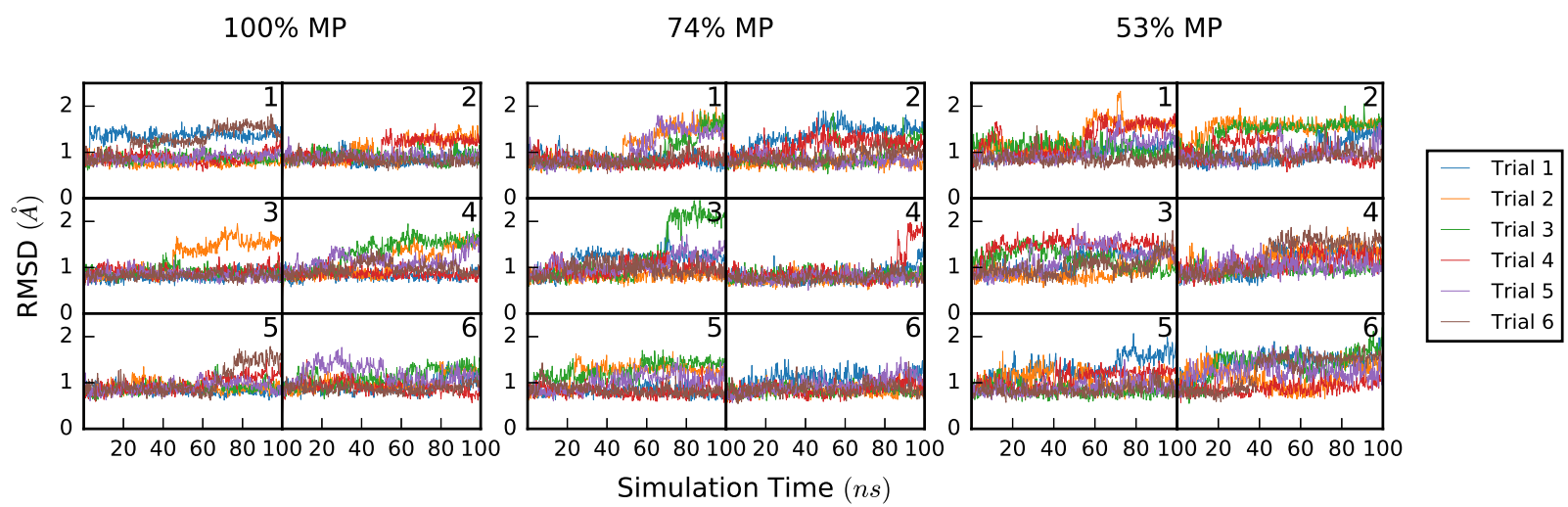

Figure S2: From left to right, RMSD of C $\alpha$ atoms for 100\%, 74\%, and 53\% MP simulations, respectively. Numbers in top right corner indicate number of unfrozen monomers.
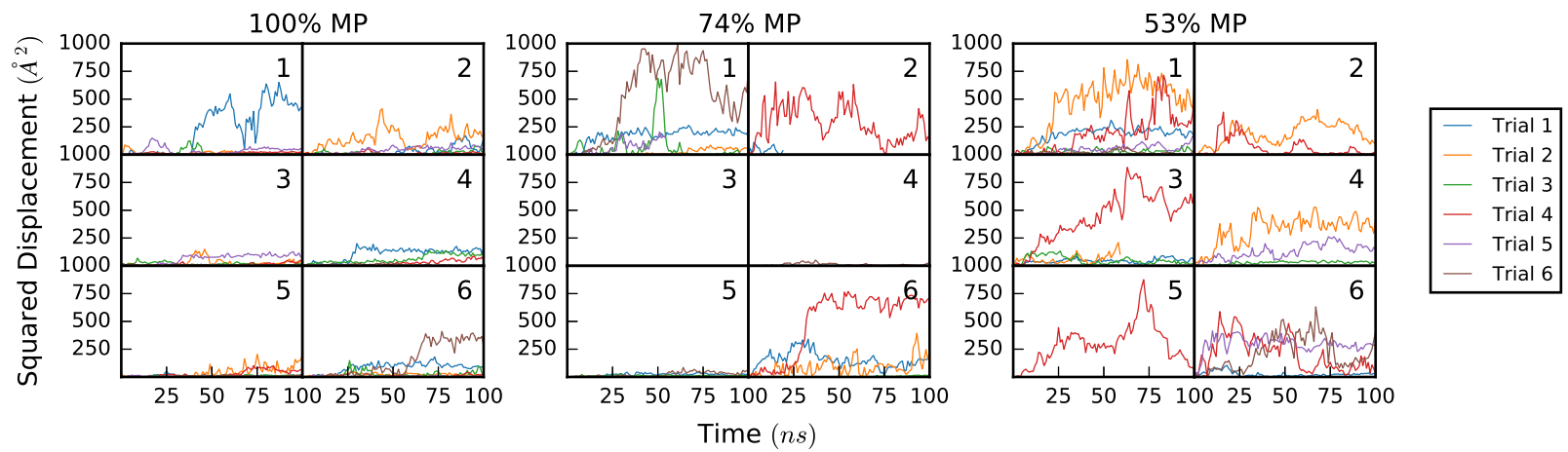

Figure S3: From left to right, displacement of adsorbed protein for $100 \%, 74 \%$, and $53 \%$ MP simulations, respectively. Numbers in top right corner indicate number of unfrozen monomers. 